\title{
The $\underline{M}$ mechanism of sulfur transfer across protein-
}

\section{protein interfaces: the CSD model system}

Francisco J. Fernández ${ }^{1}$, Ana Ardá ${ }^{1,2 \dagger}$, Miguel López-Estepa ${ }^{1}$, Juan Aranda ${ }^{3}$, Esther PeñaCon formato: Español (alfab. internacional)

Soler $^{1}$, Fernando Garces $^{4}$, Adam Round $^{5,6}$, Ramón Campos-Olivas ${ }^{7}$, Marta Bruix ${ }^{8}$, Miquel Coll $^{9,10}$, Iñaki Tuñón ${ }^{3}$, Jesús Jiménez-Barbero ${ }^{1,2 \dagger}$ and M. Cristina Vega ${ }^{1 *}$

${ }^{1}$ Chemical and Physical Biology Dpt., Center for Biological Research (CIB-CSIC), 28040 Madrid, Spain.

${ }_{1}^{2}$ Structural Biology, CIC bioGUNE, Derio-Bizkaia, Spain.

${ }^{3}$ Departamento de Química Física, Universitat de València, 46100 Burjassot, Spain.

${ }^{4}$ The Scripps Research Institute, La Jolla, 92037 California, United States of America.

${ }^{5}$ European Molecular Biology Laboratory, Grenoble Outstation, 38042 Grenoble, France.

${ }^{6}$ Unit for Virus Host-Cell Interactions, Univ. Grenoble Alpes-EMBL-CNRS, 38042 Grenoble, France.

${ }^{7}$ Spanish National Cancer Research Center (CNIO), 28029 Madrid, Spain.

${ }_{4}^{8}$ Instituto de Química Física Rocasolano (IQFR-CSIC), 28006 Madrid, Spain.

${ }^{9}$ Institut de Biologia Molecular de Barcelona (IBMB-CSIC), 08028 Barcelona, Spain.
Con formato: Español (alfab. internacional)
Con formato: Español (alfab. internacional)

Con formato: Español (alfab. internacional) 


\footnotetext{
${ }^{10}$ Institute for Biomedical Research (IRB Barcelona), 08028 Barcelona, Spain
}

KEYWORDS: CsdA-CsdE System, Cysteine desulfurase, Sulfur trafficking, Computational Chemistry, Transpersulfuration, Biogenesis.

CsdA cysteine desulfurase (the sulfur donor) and the CsdE sulfur acceptor are involved in biological sulfur trafficking and in iron-sulfur cluster assembly in the model bacterium Escherichia coli. CsdA and CsdE form a stable complex through a polar interface that includes CsdA Cys328 and CsdE Cys61, the two residues known to be involved in the sulfur transfer reaction. Although mechanisms for the transfer of a sulfur moiety across protein-protein interfaces have been proposed based on the IscS-IscU and IscS-TusA structures, the flexibility of the catalytic cysteine loops involved has precluded a high resolution view of the active-site geometry and chemical environment for sulfur transfer. Here, we have used a combination of Xray crystallography, solution NMR and SAXS, isothermal calorimetry, and computational chemistry methods to unravel how CsdA provides a specific recognition platform for CsdE and how their complex organizes a composite functional reaction environment. The X-ray structures of persulfurated $(\mathrm{CsdA})_{2}$ and persulfurated (CsdA-CsdE $)_{2}$ complexes reveal the crucial roles of the conserved active-site cysteine loop and additional catalytic residues in supporting the transpersulfuration reaction. A mechanistic view of sulfur transfer across protein-protein interfaces that underpins the requirement for the conserved cysteine loop to provide electrostatic stabilization for the in-transfer sulfur atom emerges from the analysis of the persulfurated (CsdA-CsdE $)_{2}$ complex structure. 


\section{INTRODUCTION}

Understanding key molecular recognition processes at atomic level resolution is one of the essential topics at the frontier between chemistry and biology. Cellular mobilization of elemental sulfur (S) from L-Cys is a crucial step for the biosynthesis of several vital entities, including FeS clusters, thiolated amino acids and vitamins (thiamine, biotin), lipoic acid, protein cofactors (molybdenum cofactor) and various modified RNA nucleosides. ${ }^{1,2}$ Trafficking and deployment of S for these biosynthetic pathways is accomplished by an intricate network of conserved multiprotein complexes, which act as donors and acceptors of $\mathrm{S}$ via a reactive persulfide. ${ }^{3,4}$ This exchange of $\mathrm{S}$ across protein-protein interfaces, or transpersulfuration, fulfills a range of important biological functions: it circumvents the low solubility of sulfane S, avoids the liberation of the toxic persulfide anion into the cytosol, and, importantly, allows for a more specific $\mathrm{S}$ transfer between proteins.

In Escherichia coli $(E c)$ three distinct systems can initiate sulfur transfer pathways: ISC (ironsulfur clusters), SUF (sulfur mobilization) and CSD (cysteine desulfurase) ${ }^{2,5-7}$ The ISC system comprises housekeeping proteins that are required for the constitutive assembly of Fe-S clusters and for the biosynthesis of several sulfur-containing cofactors and tRNAs. The SUF system is upregulated during $\mathrm{O}_{2}$ stress and $\mathrm{Fe}$ starvation. ${ }^{8}$ The CSD system has been implicated so far in the reconstitution of the $[4 \mathrm{Fe}-4 \mathrm{~S}] \mathrm{NadA}$ holoenzyme, ${ }^{9}$ the supply of $\mathrm{S}$ atoms for Fe-S cluster assembly via the SUF pathway, and in the biosynthesis of an unidentified sulfur-containing molecule. ${ }^{10}$ More recently, the CSD system has emerged as the likely sulfur relay system dedicated to the synthesis of cyclic $N^{\prime}$-threonylcarbamoyladenosine $\left(\mathrm{ct}^{6} \mathrm{~A}\right)$, a non-thiolated tRNA hypermodification found in bacteria, protists, fungi, and plants. ${ }^{11,12}$ In contrast to the ISC system, the mechanism of transpersulfuration in the SUF and CSD systems ${ }^{9}$ is less well understood. 
Sulfur transfer is initiated in all known systems by the persulfuration of an active site Cys residue by a pyridoxal 5'-phosphate (PLP)-dependent cysteine desulfurase (E.C. 2.8.1.7), ${ }^{13}$ IscS (ISC), ${ }^{14}$ SufS (previously known as CsdB) (SUF), ${ }^{15}$ and CsdA (CSD). ${ }^{9}$ The resultant persulfide nucleophile intermediate ${ }^{16}$ is responsible for delivering the terminal $\mathrm{S} \gamma$ atom of the persulfide to the thiol group of another reactive Cys residue in a co-substrate $\mathrm{S}$ acceptor protein, through a socalled transpersulfuration reaction (Scheme 1). It remains an open question whether the mechanisms of transpersulfuration across different cysteine desulfurases / S acceptors are as well conserved as those of the intramolecular persulfuration reaction, given the variety of interaction modes that have thus far been described. Despite CsdA is able to catalyze the S transfer from LCys to Cys358, the presence of CsdE enhances the rate of the reaction two-fold. ${ }^{9}$ Also some degree of promiscuity is observed in CsdA and cross-talk events between the CSD and SUF systems have been detected, increasing the cellular robustness under changing environmental conditions $^{9,10}$ (e.g., oxidative stress).

Despite their shared enzymatic mechanism, cysteine desulfurases are classified in two groups based on sequence and structural homology: group I (e.g., IscS) and group II (e.g., CsdA/SufS). ${ }^{13}$ In particular, group I and II cysteine desulfurases differ in the sequence of a conserved peptide motif enclosing the catalytic Cys residue (the Cys loop, ${ }^{13}{ }^{356} \mathrm{QHCAQ}^{360}$ in CsdA), whose functional relevance has hitherto remained enigmatic. The Cys loop of group I cysteine desulfurases is characterized by being highly mobile, as highlighted in the recent crystallographic studies of IscS in complex with IscU and TusA, where the mobility of the Cys loop makes the transpersulfuration possible despite the markedly different surfaces employed by IscS in these two complexes. ${ }^{17,18}$ The crystal structure of $\left(\mathrm{Fe}_{2} \mathrm{~S}_{2} \text {-(IscS-IscUD35A) }\right)_{2}$ complex from Archaeoglobus fulgidus VC-16 (Af) shows an ordered Cys loop only when the Fe-S cluster 
is being assembled. ${ }^{18}$ In contrast to IscS, the Cys loop of desulfurases from SUF and CSD systems (SufS/CsdA group II cysteine desulfurases), ${ }^{19,20}$ maintains an essentially rigid conformation. The crystal structure of $\left(\mathrm{CsdA}_{\mathrm{apo}}-\mathrm{CsdE}_{\mathrm{apo}}\right)_{2} \operatorname{complex}^{20}$ revealed that the Cys-loop remained unchanged upon CsdE binding. In this case, CsdE undergoes conformational changes in $\mathrm{H} 7$ that leads to a better fit into the active site groove of CsdA. ${ }^{20}$ Critically, the catalytic residue Cys61 from CsdE in the $\left(\mathrm{CsdA}_{\mathrm{apo}}-\mathrm{CsdE}_{\mathrm{apo}}\right)_{2}$ complex is disordered and entirely missing in the electron density map, thus limiting the chemical inferences that can be made of the interprotein S transfer. As a result, important questions such as how the chemical environment in the active site stabilizes the persulfide on its journey through different protein-protein complexes (in the CSD system or during CSD-SUF crosstalk), why the persulfuration rate is enhanced in presence of CsdE, or how sensitive the chemistry of transpersulfuration is to oxidative stress remain still unanswered.

In order to shed light into the process of transpersulfuration across the interface between $(\mathrm{CsdA})_{2}$ and CsdE, we applied biophysical, structural biology, and chemical computational approaches. We have solved the crystal structure of $(\operatorname{CsdA})_{2}$ in different persulfuration and oxidative states, and the active (persulfurated) complex of CsdA and CsdE. Furthermore, we have analyzed the CsdA-CsdE interaction in solution by NMR and compared the stability of the complexes of CsdA and CsdE and SufE with ITC. And finally, with the aid of QM/MM calculations, we propose a mechanism for the transpersulfuration reaction that is shared across group II Cys desulfurases.

\section{EXPERIMENTAL PROCEDURES}


Expression and purification of proteins. The detailed procedures used to prepare $(\operatorname{Csd} A)_{2}$ in various states including persulfurated $\left(\mathrm{CsdA}_{\mathrm{per}}\right)_{2}$ and $S$-sulfated $\left(\mathrm{CsdA}_{\mathrm{ss}}\right)_{2}, \mathrm{CsdE}$, and $\mathrm{SufE}$ are presented in the Supporting Information (SI text).

Reconstitution and purification of unmodified and persulfurated (CsdA-CsdE) ${ }_{2}$. The unmodified (CsdA-CsdE $)_{2}$ complex was prepared by mixing purified components, $(\mathrm{CsdA})_{2}$ (obtained from His $_{6}$-CsdA after proteolytic removal of the histidine tag with TEV protease) and $\mathrm{CsdE}^{-\mathrm{His}_{6}}$, at a 1:1.5 molar ratio for $30 \mathrm{~min}$ at $277 \mathrm{~K}$, and subjecting the mixture to gel filtration chromatography on a Superdex 200 10/300GL (GE Healthcare) in 10 mM HEPES pH 7.4, 50 $\mathrm{mM} \mathrm{NaCl}, 0.1 \mathrm{mM}$ EDTA. Peak fractions were concentrated to $20 \mathrm{mg} / \mathrm{ml}$ using $10-\mathrm{kDa}$ ultrafiltration devices (Millipore) and stored frozen at $193 \mathrm{~K}$. The persulfurated (CsdA $\mathrm{per}^{-}$ $\left.\mathrm{CsdE}_{\mathrm{per}}\right)_{2}$ complex was prepared by incubating the unmodified (CsdA-CsdE) $)_{2}$ complex with 5 $\mathrm{mM} \mathrm{L}$-cysteine for $30 \mathrm{~min}$ at $298 \mathrm{~K}$ and then separating the persulfurated $\left(\mathrm{CsdA}_{\text {per }}-\mathrm{CsdE}_{\mathrm{per}}\right)_{2}$ complex from excess CsdE-His 6 , L-cysteine, and L-alanine, by gel filtration (as indicated above). The $\left(\mathrm{CsdA}_{\mathrm{per}}-\mathrm{CsdE}_{\mathrm{per}}\right)_{2}$ complex was concentrated and stored as described for the unmodified (CsdA-CsdE $)_{2}$ complex.

Crystallization and structure determination of $(\mathrm{CsdA})_{2}$ variants. Crystallization, X-ray data collection, structure determination, and refinement of $(\mathrm{CsdA})_{2}$, persulfurated $\left(\mathrm{CsdA}_{\text {per }}\right)_{2}$ (Figure S1), and $S$-sulfated (CsdA), are described in detail in the SI. Complete data processing and refinement statistics are given in Table 1 and Table S1.

Crystallization of persulfurated (CsdA-CsdE $)_{2}$. Persulfurated $\left(\mathrm{CsdA}_{\mathrm{per}}-\mathrm{CsdE}_{\mathrm{per}}\right)_{2}$ complex was crystallized by sitting-drop vapor diffusion using $1 \mu \mathrm{l}$ complex and $1 \mu 1$ reservoir condition (0.1 M Tris- $\mathrm{HCl}(\mathrm{pH} 8.5), 0.1 \mathrm{M}$ magnesium acetate, 10\% (w/v) PEG 8000) at $293 \mathrm{~K}$. Prismatic crystals with an intense yellow color developed in 1-4 d, reaching final dimensions of $\sim 80 \times 20 \times$ 
$20 \mu \mathrm{m}$. Prior to diffraction data collection, crystals were equilibrated in crystallization condition and $30 \%(\mathrm{v} / \mathrm{v})$ glycerol, then mounted in nylon loops, and flash-frozen in liquid nitrogen.

X-Ray data collection. A complete data set consisting of 720 images at $0.5^{\circ}$-oscillation angle $\left(360^{\circ}\right.$ in total) was collected on ID23-1 beamline (ESRF, Grenoble, France), equipped with an ADSC Q310r detector, at $100 K$ and using a crystal-to-detector distance of $253 \mathrm{~mm}$. Persulfurated $\left(\mathrm{CsdA}_{\text {per- }}-\mathrm{CsdE}_{\mathrm{per}}\right)_{2}$ crystals belonged to the $P 2{ }_{1} 2_{1} 2_{1}$ orthorhombic space group with cell dimensions $a=74.86 \AA, b=115.14 \AA$, and $c=604.97 \AA$, and diffracted to $2.49-\AA$ resolution. The asymmetric unit contained four biological heterotetramers, thereby yielding 8 independent $\mathrm{CsdA}_{\text {per }}-\mathrm{CsdE}_{\text {per }}$ heterodimers/interfaces. The data set was processed with $\mathrm{XDS}^{21}$ and scaled with Aimless. ${ }^{22}$ Complete data processing statistics are given in Table 1.

Structure determination and refinement. The crystal structures of $\left(\mathrm{CsdA}_{\mathrm{per}}\right)_{2}$ at $1.76 \AA$ resolution $\left(\mathrm{R} / \mathrm{R}_{\text {free }}\right.$ values of $\left.0.166 / 0.224\right)$ (Table 1 and Figure $\left.\mathrm{S} 1\right)$ and $S$-sulfated $\left(\mathrm{CsdA}_{\mathrm{ss}}\right)_{2}$ at $2.05 \AA$ resolution $\left(\mathrm{R} / \mathrm{R}_{\text {free }}\right.$ values of 0.154 / 0.190) (Table S1) were determined by molecular replacement using as search model a structure of a new $(\mathrm{CsdA})_{2}$ crystal structure $\left(1.99 \AA, \mathrm{R} / \mathrm{R}_{\text {free }}\right.$ values of $0.137 / 0.191$ ) solved independently by selenomethionine (Se)-SAD (SI text, Table S1). The structure of the persulfurated $\left(\mathrm{CsdA}_{\mathrm{per}}-\mathrm{CsdE}_{\mathrm{per}}\right)_{2}$ complex was determined by molecular replacement with PHASER ${ }^{23}$ using as search models the crystal structures of $(\mathrm{CsdA})_{2}$ (PDB 5FT4) and CsdE (PDB 5FT7) simultaneously during the rotation and translation searches. The molecular replacement solution was then completed manually through cycles of maximum likelihood refinement in phenix.refine ${ }^{24}$ interspersed with manual building and validation in $\mathrm{Coot}^{25}$ and MolProbity. ${ }^{26}$ Refinement statistics are in Table 1. Atomic coordinates and structure factors of persulfurated ( $\left.\mathrm{CsdA}_{\text {per }}-\mathrm{CsdE}_{\mathrm{per}}\right)_{2}$ have been deposited with PDB 5FT8. 


\section{Protein expression and purification of uniformly labeled $\left[\mathrm{U}_{-}{ }^{13} \mathrm{C},{ }^{15} \mathrm{~N}\right]-\mathrm{CsdE}-\mathrm{His}_{6}$.}

Uniformly labeled $\left[\mathrm{U}-{ }^{13} \mathrm{C},{ }^{15} \mathrm{~N}\right]-\mathrm{CsdE}_{-} \mathrm{His}_{6}$ for nuclear magnetic resonance studies was produced by growing E. coli BL21(DE3) bacteria transformed with pET3a-CsdE-His 6 in minimal medium using [U-15N]-ammonium sulfate and $\left[\mathrm{U}-{ }^{13} \mathrm{C}\right]-\mathrm{D}$-glucose as sole sources of nitrogen and carbon, respectively. Purification was carried out as indicated for non-labeled CsdE-His 6 without modifications.

CsdA-binding surface of CsdE-His $_{6}$ assessed by NMR. Proteins used in titration experiments, $\left[\mathrm{U}_{-}{ }^{13} \mathrm{C},{ }^{15} \mathrm{~N}\right]-\mathrm{CsdE}_{-} \mathrm{His}_{6}$ and unlabeled $\mathrm{CsdA}$, were dissolved in $50 \mathrm{mM}$ potassium phosphate ( $\mathrm{pH} 7.5$ ), $0.1 \mathrm{mM}$ EDTA, in $90 \% / 10 \% \mathrm{H}_{2} \mathrm{O} / \mathrm{D}_{2} \mathrm{O}$. NMR experiments were acquired at $298 \mathrm{~K}$ on a Bruker Avance $600 \mathrm{MHz}$ spectrometer equipped with a cryoprobe for 1D and 2D, and on a Bruker Avance $800 \mathrm{MHz}$ for 3D. The spectra were processed with TopSpin 2.1 (Bruker), and 2D and 3D spectra were analyzed with the program CCPNmr Analysis. ${ }^{27} 1 \mathrm{D}{ }^{1} \mathrm{H}-$ ${ }^{13} \mathrm{C}$ and ${ }^{1} \mathrm{H}-{ }^{15} \mathrm{~N}$ HSQC spectra (5120 and 10240 scans respectively), $2 \mathrm{D}{ }^{1} \mathrm{H}_{-}{ }^{15} \mathrm{~N}$ HSQC spectra (3072 data points in t2, 128 in t1, 96 scans), and 3D spectra (2048 data points in t3, 30 in t2 $\left({ }^{15} \mathrm{~N}\right)$, and 128 in $\mathrm{t} 1\left({ }^{13} \mathrm{C}\right), 32$ scans) were acquired using standard Bruker pulse sequences. 1D heteronuclear spectra were processed with a line broadening of $10 \mathrm{~Hz}$ and $20 \mathrm{~Hz}\left({ }^{1} \mathrm{H}^{13} \mathrm{C}^{13}\right.$ and ${ }^{1} \mathrm{H}-$ ${ }^{15} \mathrm{~N}$ HSQC respectively). ${ }^{1} \mathrm{H}_{-}{ }^{15} \mathrm{~N}$ backbone resonance assignment for $\left[\mathrm{U}-{ }^{13} \mathrm{C},{ }^{15} \mathrm{~N}\right]-\mathrm{CsdE}-\mathrm{His}_{6}$ was performed using $\mathrm{HSQC}, \mathrm{CBCANH}^{28}$ and $\mathrm{CBCA}(\mathrm{CO}) \mathrm{NH}^{29}$ spectra. Most $\mathrm{NH} / \mathrm{Ha}$ crosspeaks could be assigned and were in agreement with published assignments (BMRB code: 5630 ). ${ }^{30}$

The interaction between CsdA and $\left[\mathrm{U}_{-}{ }^{13} \mathrm{C},{ }^{15} \mathrm{~N}\right]$-CsdE-His 6 was probed by stepwise addition of unlabeled CsdA to a $0.4 \mathrm{mM}\left[\mathrm{U}_{-}{ }^{13} \mathrm{C},{ }^{15} \mathrm{~N}\right]-\mathrm{CsdE}-\mathrm{His}_{6}$. At each titration point $(1: 0,1: 0.2,1: 0.4$, and 1:0.6), ${ }^{1} \mathrm{H}-{ }^{15} \mathrm{~N}$ HSQC spectra were recorded, and the rate of intensity loss was obtained by fitting the volume of every $\mathrm{NH} / \mathrm{Ha}$ crosspeak at different ratios to an exponential decay function 
$V=A \mathrm{e}^{-B x}$, where $V$ is the volume of an $\mathrm{NH} / \mathrm{Ha}$ crosspeak, $x$ is the CsdE/CsdA molar ratio, and $A$ and $B$ are fitted parameters.

Computational studies. Protein modeling and quantum mechanical / molecular mechanical (QM/MM) calculations were performed with the programs AMBER,${ }^{31}$ NAMD,${ }^{32}$ fDynamo, ${ }^{33}$ and Gaussian09. ${ }^{34}$ We constructed a model that was derived from the crystal structure of persulfurated $\left(\mathrm{CsdA}_{\text {per- }}-\mathrm{CsdE}_{\mathrm{per}}\right)_{2}$ complex, with CsdE Cys61 modeled with a thiolate side chain. The QM subsystem consisted of the side chain of the persulfurated CsdA Cys358, the side chains of the CsdE Cys61 and CsdA His119 and His120, and the Lys222-PLP cofactor (69 atoms in total). The QM subsystem was described at the M06-2X/6-31G**35 level of theory while the MM system was described using the AMBER force field as implemented in fDynamo. ${ }^{36,37}$ To saturate the valence of the $\mathrm{QM} / \mathrm{MM}$ boundary we used the link atom procedure. ${ }^{38,39}$ Additional information on the computational chemistry methodology is presented in the SI Text.

\section{RESULTS AND DISCUSSION}

The interaction between the CsdA homodimer and each CsdE monomer is non-cooperative and is more stable than in the complexes (SufS-SufE $)_{2}$ or (CsdA-SufE) $)_{2}$. We performed ITC titration studies to determine the thermodynamic constants of the interaction between CsdA and CsdE (Figure 1). The results showed a thermodynamically (both enthalpically and entropically) favorable curve with an expected 1:1 stoichiometry $(n=1.072)$ and a $K_{\mathrm{D}} \sim 117 \mathrm{nM}(\Delta \mathrm{G}=-9.5$ $\mathrm{kcal} / \mathrm{mol}$ at $298 \mathrm{~K}$ ) (Figure $1 \mathrm{~A}$, SI text, Table S2, and Figure S2). In contrast to the binding isotherms of IscS-IscU ${ }^{40}$ and SufS-SufE, ${ }^{41,42}$ which are biphasic with exothermic and endothermic phases, the ITC binding isotherm for the CsdA-CsdE interaction is in agreement with a monophasic and exothermic interaction. This difference suggests that the CsdA-CsdE 
interaction is non-cooperative and, in particular, does not exhibit the flip-flop allosteric regulation mechanism described for other desulfurase-acceptor pairs.

The thermodynamic data obtained indicate that the (CsdA-CsdE $)_{2}$ complex exists as a more stable complex than the homologous (SufS-SufE) $)_{2}$ complex $^{41}$ and related complexes, like (IscS$\mathrm{IscU}_{2},{ }^{40,43}$ which have a 10-20-fold weaker affinity. Interestingly, ITC measurements of SufS ${ }_{\text {apo }}$ interaction with alkylated $\mathrm{SufE}_{\text {alk }}$ (prepared by incubation of $\mathrm{SufE}_{\text {apo }}$ with iodoacetamide, which quantitatively alkylates the reactive Cys51) show a 10-fold tighter $K_{\mathrm{D}}(260 \mathrm{nM})$ than the $\mathrm{SufS}_{\text {apo- }}$ SufE $_{\text {apo }}$ interaction and a monophasic behavior. ${ }^{41}$ The $\mathrm{SufS}_{\text {apo }}-\mathrm{SufE}_{\text {alk }}$ interaction therefore might approximate the CsdA-CsdE interaction more closely. Since the peptide containing the alkylated Cys51 in SufE $E_{\text {alk }}$ is more solvent exposed than the unmodified Cys51, where the same peptide is protected from solvent in a hydrophobic pocket, the structure around Cys51 in SufE alk might adopt a similar conformation to that of CsdE. If this were the case, the tigther $K_{\mathrm{D}}$ for $\mathrm{SufS}_{\text {apo- }}$ SufE $_{\text {alk }}$ could be the result of the formation of a complex structurally analogous to CsdA-CsdE.

CsdA is also known to interact with SufE in a crosstalk between the CSD and SUF systems, though less tightly than with CsdE. ${ }^{9}$ We did not detect measurable binding of CsdA and $\mathrm{SufE}_{\text {apo }}$ by ITC (data not shown), indicating that their association constant is very weak (below mM range). Despite the absence of a stable (CsdA-SufE) $)_{2}$ complex, CsdA can persulfurate SufE in vitro in a quantitative fashion, indicating that a short-lived catalytic (CsdA-SufE) $)_{2}$ complex may exist. This observation is compatible with the reported role of CsdA in Fe-S biogenesis by recruiting the SufBCD complex and SufE. ${ }^{10}$

The composite active site of CsdA is preformed for persulfuration: Cys358 $\mathrm{S} \gamma$ in $\left(\operatorname{Csd} A_{\text {per }}\right)_{2}$ is stabilized by residues from both subunits. To investigate the structural and chemical consequences of the persulfuration of CsdA we solved the crystal structures of 
persulfurated CsdA and of the oxidized $S$-sulfated $\operatorname{CsdA},\left(\operatorname{CsdA}_{\mathrm{ss}}\right)_{2}$ at $1.76 \AA$ and $2.05 \AA$ resolution, respectively. The two covalently modified CsdA structures showed no significant differences in their fold arrangement (Figure S1). CsdA belongs to the fold-type I class V aminotransferase or $\alpha$-family of PLP-dependent enzymes, ${ }^{17,40}$ which are structurally characterized by a constitutive dimer built from identical two-domain subunits (green and violet, Figure $1 \mathrm{~A}$ ). The covalently modified Cys358 is at the tip of the so-called Cys loop motif in the small domain (residues 4-22 and 294-401) (Figure 1 B and Figure 1 C). ${ }^{13}$ The large domain (residues 23-293) harbors the PLP cofactor and the cysteine-substrate binding pocket. In both crystal structures, easily recognizable electron density indicated the presence of the PLP cofactor as an internal aldimine covalently bound to Lys222 (Figure $1 \mathrm{C}$ and Figure 2).

In $\left(\mathrm{CsdA}_{\text {per }}\right)_{2}$, the persulfido moiety on Cys358 is stabilized by hydrogen bonding interactions with key residues, including the imidazole side chain of His119 and the main-chain amide of Lys $250^{*}$ from the other subunit, which also participate in stabilizing the active site during transpersulfuration (Figures $1 \mathrm{C}$ and $2 \mathrm{~A}$ ). The hydrogen bond network centered on the persulfidic S $\delta$ atom holds the Cys358 modified side chain already in an orientation that is ready for reaction, therefore priming CsdA for transpersulfuration. The dimer interface in $\operatorname{Csd}_{\text {per }}$ involves extensive contacts between residues from the large domain and the small domain from the opposite subunit at the rim of the active-site entryway, thereby leaving a large accessible area for substrates and interaction partners. When the structure of $\mathrm{CsdA}_{\text {per }}$ is compared with the structure of SufS (PDB 1JF9) ${ }^{19}$ it becomes apparent that several N-terminal motifs in SufS have been removed or reshaped in CsdA to increase access to the active site, including the deletion of the residues forming a $\beta$-hairpin in SufS (residues 18-22) and the bending of the long $\mathrm{H} 3$ helix at the dimer interface after a $45^{\circ}$ turn at Glu66-Ala67. 
Further oxidative modification of persulfurated Cys358 by dissolved $\mathrm{O}_{2}$ leads to the formation of an $S$-sulfation modification that cannot be repaired in bacteria, and which is unambiguously observed in the structure of $\left(\mathrm{CsdA}_{\mathrm{ss}}\right)_{2}$ (Figure $\left.2 \mathrm{~B}\right)$. The reactive nature of persulfurated Cys358 is underscored by the fast conversion to the $S$-sulfated modification by exposure to air. The bulkier $S$-sulfate group is easily accommodated into the wide active site by the same residues (see below) that stabilize the persulfuration modification thereby impairing the $\mathrm{S}$ transfer process to any downstream acceptor (CSD or SUF system). The ease with which the $S$-sulfation of persulfurated Cys358 occurs by exposure to air agrees with the fact that a functional CSD system would be impaired during oxidative stress. ${ }^{44}$

Crystal structure of the persulfurated (Csd $\left.\mathrm{A}_{\text {per }}-\mathrm{CsdE}_{\mathrm{per}}\right)_{2}$ complex. To gain insight into the structural basis and the mechanism by which persulfuration occurs across the interface between cysteine desulfurases and their sulfur acceptor co-substrates, we determined the $2.49 \AA$ resolution X-ray crystal structure of the persulfurated $\left(\mathrm{CsdA}_{\mathrm{per}}-\mathrm{CsdE}_{\mathrm{per}}\right)_{2}$ complex from Escherichia coli. The asymmetric unit of the $\left(\mathrm{CsdA}_{\mathrm{per}}-\mathrm{CsdE}_{\mathrm{per}}\right)_{2}$ crystal contained four independent heterotetramers with a solvent content of $54 \%$. Three heterotetramers were well ordered in the crystal and served as the basis for our analyses; the fourth tetramer suffered from static disorder and was not considered further. The heterocomplex is formed by two subunits of $\mathrm{CsdE}_{\text {per }}$ bound to each active site of the $\mathrm{CsdA}_{\text {per }}$ homodimer, with the small helical domain burying a surface of $750 \AA^{2}$. The co-substrate CsdE, the S acceptor in the CSD system, showed a fold consisting of a two-layered $\alpha / \beta$ sandwich with a core three-stranded $\beta$-sheet surrounded by a bundle of five $\alpha$-helices (yellow and blue, Figure 1 A). The crystal structures of CsdA, CsdE, and the heterodimeric complex were in complete agreement with the corresponding structures in solution as judged by small-angle X-ray scattering data (SAXS, SI Text and Figure S3). 
The $\mathrm{CsdA}_{\text {per }}-\mathrm{CsdE}_{\text {per }}$ interface comprises helices $\mathrm{H} 16$ and $\mathrm{H} 18$ from CsdA $\mathrm{A}_{\text {per }}$ and helices $\mathrm{H} 2$ and $\mathrm{H} 4$ and the loop around the acceptor Cys61 from $\mathrm{CsdE}_{\text {per }}$ (Figure $3 \mathrm{~A}$ ). While CsdA remains essentially unchanged in the persulfurated complex, the structure of bound $\mathrm{CsdE}_{\text {per }}$ undergoes conformational rearrangements that affect the loop connecting helices $\mathrm{H} 6-\mathrm{H} 7$ and the $\alpha$-helix $\mathrm{H} 7$ of the free form of CsdE. In bound $\mathrm{CsdE}_{\text {per, }}$, the H6-H7 loop becomes a $\alpha$-helix (new H7) and the free conformation of the old $\mathrm{H} 7$ becomes distorted into two helical segments, H8' (a 310 helix) and $\mathrm{H} 8$, a shortened C-terminal $\alpha$-helix (Figure $3 \mathrm{~A}$ ). The concerted structural changes help to maintain the spacing and the $85^{\circ}$ tilt between helices $\mathrm{H} 7$ and $\mathrm{H} 8$ in the bound conformation of $\operatorname{CsdE}_{\text {per }}$ (Figure 3, blue arrow). Furthermore, this conformational change allows the assembly of a catalytically active complex by pushing the acceptor Cys61 to a position closer to the donor Cys358 (11.8 $\AA$ between the $\mathrm{S} \gamma$ atoms) with respect to the free form of CsdE (Figure 3 A, grey arrow). In the $\left(\mathrm{CsdA}_{\text {per }}-\mathrm{CsdE}_{\mathrm{per}}\right)_{2}$ crystal structure the two persulfidic S $\delta$ atoms, which can be unambiguously traced in electron density maps (Figure $2 \mathrm{C}$ ), are separated merely by a $\sim 4.5 \AA$ gap.

The persulfurated $\left(\mathrm{CsdA}_{\text {per }}-\mathrm{CsdE}_{\mathrm{per}}\right)_{2}$ structure also underscores the dynamic nature of the CsdA-CsdE interaction. When six independent heterodimers are superimposed using only the CsdA coordinates, only the CsdE Cys61 tip residues adopt a fixed position and orientation, while the complete $\mathrm{CsdE}_{\text {per }}$ subunit swings about a hinge axis defined by $\mathrm{CsdA}_{\text {per }} \alpha$-helix H16 (residues 338-349) with an angular spread of $8^{\circ}$ (Figure S4). This degree of structural plasticity in both CsdE and in the CsdA-CsdE interface might facilitate the efficient transfer of CsdA Cys358S $\delta$ to CsdE Cys61S $\gamma$ by allowing the motif harboring the acceptor Cys61 side chain to reach deep enough into the more rigid active site of persulfurated CsdA. 
The solution CsdA-CsdE interface recapitulates the persulfurated interface. The mutual orientation and overall interactions between $\mathrm{CsdA}_{\text {per }}$ and $\mathrm{CsdE}_{\text {per }}$ observed in the crystal is also observed in in the apo complex in solution by NMR techniques. Spectral perturbations where observed that allowed mapping the behavior of specific residues onto the protein-protein interface. In particular, we used uniformly ${ }^{15} \mathrm{~N}$ labeled CsdE that was titrated with unlabeled CsdA up to a 1:0.6 molar ratio. Most NH crosspeaks could be assigned and agreed with the previously published assignments. ${ }^{30}{ }^{1} \mathrm{H}-{ }^{15} \mathrm{~N}$ HSQC spectra acquired for every titration point (Figure $4 \mathrm{~A}$ ) revealed a sharp decrease in the integrated peak intensity for all the backbone NH CsdE correlations upon CsdA addition, while the intensity of the crosspeaks corresponding to the amide side chains were far less perturbed. These observations corroborate the formation of a high affinity (CsdA-CsdE $)_{2}$ complex in solution. Fitting the decreasing volume of the $\mathrm{NH}$ crosspeaks to an exponential decay function, we identified the CsdE residues mostly affected by CsdA binding: Tyr34, Arg35, Glu36, Cys61, Leu67, Glu84, Val88, Arg89, Gly90, Leu92, Ala93, Va194, Leu96, Arg122 and Ser130 (Figure 4 B,C). These residues map onto the three $\alpha$-helices of CsdE that lie at the interface with CsdA, therefore delineating the CsdA-CsdE solution interface (Figure 4 B). Fittingly, this is in perfect agreement with the interface observed in the crystallographic structure.

\section{Stabilization of the transpersulfuration reaction between CsdA and CsdE requires the} canonical sequence motif of group II Cys desulfurases. The composite active site revealed by the crystal structure of persulfurated $\left(\mathrm{CsdA}_{\mathrm{per}}-\mathrm{CsdE}_{\mathrm{per}}\right)_{2}$ had important consequences for the mechanism of transpersulfuration. As in the case of the intra-molecular persulfuration of Cys358 by L-Cys, the mechanism for the inter-protein sulfur transfer reaction can be proposed to consist in a nucleophilic attack by the deprotonated $\mathrm{CsdE}_{\text {apo }} \mathrm{Cys} 61 \mathrm{~S} \gamma$ onto the persulfurated $\mathrm{CsdA}_{\text {per }}$ 
Cys358S 8 . A preexisting $\mathrm{CsdE}_{\text {apo }}$ Cys61S $\gamma$ thiolate is assumed based on the experimentally determined $\mathrm{p} K_{a}$ for Cys61 of 6.5 , a value that is nearly two $\mathrm{pH}$ units lower than that of free cysteine and ensures a predominantly ionized thiolate $(>88 \%) .{ }^{44}$ Likewise, the measured $\mathrm{p} K_{a}$ value for the equivalent Cys51 in the sequence and structurally homologous SufE is 6.3 and is therefore consistent with the parallel functional roles played by CsdE and SufE as recipients of persulfidic $\mathrm{S}$ atoms. The postulated nucleophilic attack requires the two sulfur atoms to be within a $4.5 \AA$ distance, which appeared difficult to reconcile with the inter-S $\gamma$ distances observed in the available crystal structure of the unmodified complex,${ }^{20}$ where no obvious interactions were found to stabilize a productive reaction intermediate. In the persulfurated $\left(\mathrm{CsdA}_{\mathrm{per}}-\mathrm{CsdE}_{\mathrm{per}}\right)_{2}$ structure reported here, however, a stabilizing chemical environment can be observed as a result of a productive CsdA-CsdE binding configuration. The optimal geometry for transpersulfuration requires the reorientation of the reactive Cys side chains to align Cys61 C $\beta-S \gamma$ and Cys358 S $\gamma-$ S $\delta$ bonds, in order to bring the thiol sulfur atoms into closer proximity. This configuration is compatible with the structure of the active site observed in the $\left(\mathrm{CsdA}_{\mathrm{per}}-\mathrm{CsdE}_{\mathrm{per}}\right)_{2}$ complex (Figure 2 C). The Cys358 persulfide of CsdA is stabilized by His $120 \mathrm{~N} \delta \square \square$ Lys $250 *$ from the other subunit, and Cys358's own backbone amide atoms through a water-mediated contact (Figure 2 A,C). As expected, these interactions with Cys358 $\mathrm{S} \delta-\mathrm{S} \gamma$ are also observed in the functional persulfurated $\left(\mathrm{CsdA}_{\text {per }}\right)_{2}$ active site in presence of CsdE (Figure $2 \mathrm{~A}$ ). In the persulfurated complex, $\mathrm{CsdA}_{\text {per }}$ Cys358 loop residues stabilize $\mathrm{CsdE}_{\mathrm{per}} \mathrm{Cys} 61 \mathrm{~S} \gamma$ and $\mathrm{S} \delta$ atoms via direct and water-mediated hydrogen bonds with the backbone NH groups of Gln356, His357, and Cys358 (Figure 2 C). Glu62 is the only other residue from the Cys61 hairpin that interacts with CsdA, specifically through the Glu62 carboxylate group and Glu62O to the carbonyl Ala354O and to His338Ne2 respectively. This hydrogen bond network provides the chemical 
rationale for the conservation of the amino acid sequence in the Cys358 loop of CsdA $\left({ }^{356} \mathrm{QHCAQ}^{360}\right)$, and, conceivably, it can be extended to the other class II cysteine desulfurases where this sequence signature is conserved, including SufS. ${ }^{13}$ The fully oxidized state of persulfurated Cys358 S $\gamma-\mathrm{S} \delta$ impairs the reactivity of the sulfur atom by blocking the $\mathrm{S}$ transfer (Figure 2 B).

\section{Residues from CsdA Cys-loop would further assist catalysis by stabilizing the attacking}

CsdE Cys61 thiolate. The $\operatorname{CsdA}_{\text {per- }}-\operatorname{CsdE}_{\text {per }}$ interface creates the optimum environment for the relocation of Cys61S $\gamma$ in a favorable orientation for transpersulfuration. To shed light into the mechanism of the transpersulfuration reaction, we resorted to computational chemistry calculations using the persulfurated $\left(\mathrm{CsdA}_{\text {per }}-\mathrm{CsdE}_{\mathrm{per}}\right)_{2}$ complex structure as the template and removing Cys61S from CsdE (Figure 5). We performed molecular dynamics (MD) simulations with the AMBER 99SB force field for the $\left(\mathrm{CsdA}_{\text {per }}-\mathrm{CsdE}\right)_{2}$ complex where the Cys61 was modeled as a thiolate (see the SI Text and Figures S5 and S6 for details). The total simulation time was 50 ns. Afterwards, the system was minimized by means of the hybrid Quantum Mechanics/Molecular Mechanics (QM/MM) methodology at the M06-2X/6-31G**/MM level of theory, where the QM subsystem was composed of the side chains of Cys61, Cys358, His119, His 120, and the PLP cofactor (69 atoms in total). The resulting structure for the active site (Table S3) is depicted in Figure 5 B.

Analysis of the MD simulation shows that the CsdE subunit has an important flexibility, according to the previous observations. The $\mathrm{S} \delta$-S $\gamma$ distance fluctuates between 3.5-8.5 $\AA$ (Figure 6), a distance variation that can be accounted for by the inherent pivoting of CsdE over the CsdA active site groove observed in the crystal structure of the persulfurated complex (Figure S4). Thus, during the $50 \mathrm{~ns}$ trajectory the system visited conformations where the direct transfer from 
$\mathrm{CsdA}_{\mathrm{per}} \mathrm{Cys} 358 \mathrm{~S} \delta$ to $\mathrm{CsdE}_{\text {apo }}$ Cys61S $\gamma$ is feasible. On the contrary, equivalent MD simulations of the system with a protonated CsdE Cys61 shows a substantially larger S $\delta$-S $\gamma$ distance in which direct transpersulfuration is not possible (Figure S6). This result confirms that Cys61 must be deprotonated in order to favor the process.

The QM/MM optimized $\left(\mathrm{CsdA}_{\mathrm{per}}-\mathrm{CsdE}\right)_{2}$ complex exhibited a reaction-competent geometry for the thiol-persulfido exchange reaction between $\mathrm{CsdA}_{\text {per }} \mathrm{Cys} 358 \mathrm{~S} \delta$ and $\mathrm{CsdE}_{\text {apo }}$ Cys61S $\gamma$. The Cys61S $\gamma$ atom is found at $3.7 \AA$ from the persulfurated Cys358S $\delta$, thereby suggesting the possibility of a direct nucleophilic attack, which could lead to the persulfuration of CsdE. The position and orientation of CsdE Cys61 is favored by the hydrogen bond network established with the backbone NH groups of CsdA Gln356 and Cys358. In turn, persulfurated Cys358 from CsdA is kept by means of hydrogen bonds to the imidazole rings of His119 and His120 and the backbone amide group of Lys $250^{*}$. The positive polarization generated by the Cys358-loop backbone structure, specifically through the electrostatic stabilization afforded by the aligned amide NH functions of Gln356 and Cys358 from the Cys loop, would further assist catalysis by stabilizing the Cys61 thiolate over the corresponding thiol and/or the negative charge spread over the transition state.

To gain further insight into the enzymatic mechanism of the transpersulfuration, we explored the Potential Energy Surface (PES) along the antisymmetric stretch coordinate $\mathrm{S} \delta$-S $\gamma$-S $\delta$ that delineates the pathway for inter-protein $\mathrm{S}$ transfer. We found a transition structure with a potential energy barrier of $20.2 \mathrm{kcal} \mathrm{mol}^{-1}$. This structure is presented in Figure 7 and shows the protonated S $\gamma$ placed midway between the S $\delta$ atoms of CsdE Cys61 (2.4 $\AA$ ) and CsdA Cys358 $(2.9 \AA)$. According to this structure the nucleophilic attack of the Cys61 thiolate on the persulfurated Cys358 is favored by the hydrogen bond interactions of the side chains of His119 
and Lys $250^{*}$ with the S $\gamma$ atom of Cys 358 and by the electrostatic stabilization provided by PLP. In this scenario, the CsdA Cys358 thiolate formally developed after the direct S transfer to the acceptor CsdE Cys61 would be reprotonated by His 119 and/or Lys250*. Of the two residues, Lys $250^{*}$, by virtue of being highly exposed to the bulk solvent, could easily reprotonate the temporary thiolate generated on Cys $358 \delta$ by taking up protons from the solvent.

The role of His 119 , His 120 and Lys $250 *$ in mediating the transpersulfuration could be extrapolated to other sulfur donor-acceptor pairs since they are either conserved in SufS (His123, His124) or substituted by a potentially isofunctional group (His55). In IscS, the equivalent residues can be identified from the superposition of the CsdA and IscS in complex with their S acceptors; His343 in IscS could take the place of His119 and/or His120 in providing stabilization to the catalytic Cys321, while there is no clear counterpart to Lys250* in CsdA except for IscS Lys99, whose terminal ammonium side chain could potentially substitute for Lys $250^{*}$ amide main-chain function. The conservation of functional roles across cysteine desulfurases suggests that the fundamental chemical reaction underlying the transfer of $\mathrm{S}$ atoms across protein-protein interfaces could be essentially conserved across desulfurases in spite of considerable sequence divergence. Furthermore, the sequence variation at His119, His120 and Lys250* positions could be partly responsible for the greater efficiency of CsdA as a cysteine desulfurase enzyme when compared with SufS/IscS. ${ }^{9}$ If true, the conservation of the active site structure (Cys loop) plus the tunability of additional catalytic residues (e.g., His119, Lys250*) would underscore the conservation of the mechanistic processes of the transpersulfuration reaction.

\section{CONCLUSION}

In conclusion, the sequence and structural similarities between CsdA/CsdE and SufS/SufE suggest the existence of a common sulfur acceptor-donor recognition mechanism shared by the 
CSD and SUF systems. The conformational changes and intersubunit adjustments observed in the persulfurated $\left(\mathrm{CsdA}_{\mathrm{per}}-\mathrm{CsdE}_{\mathrm{per}}\right)_{2}$ complex result in a tighter and more extensive interface than in a previous report ${ }^{20}$ (SI text). In the persulfurated $\left(\mathrm{CsdA}_{\text {per- }}-\mathrm{CsdE}_{\mathrm{per}}\right)_{2}$ interface, the distance between the reactive persulfide and thiol groups $\left(\mathrm{CsdA}_{\text {per }} \mathrm{Cys} 358 \mathrm{~S} \delta\right.$ and $\left.\mathrm{CsdE}_{\mathrm{per}} \mathrm{Cys61S \gamma}\right)$ becomes $0.5-1 \AA$ shorter than in the unmodified complex, reaching a more favorable position and orientation for transpersulfuration (Figures $1 \mathrm{~B}$ and $5 \mathrm{~A}$ ). Since in the (CsdA-CsdE) $)_{2}$ complexes (persulfurated and unmodified) the access to the catalytic center is unimpeded, CsdA can achieve an essentially continuous persulfurated state, whereby transpersulfuration of CsdE would be closely followed by the re-persulfuration of CsdA Cys358. In keeping with the modest effect of CsdE in CsdA desulfurase activity (a 2-fold increase ${ }^{9}$ ), the role of CsdE in enhancing CsdA activity could be limited to provide an acceptor Cys for Cys358 persulfide. The MD simulations and $\mathrm{QM} / \mathrm{MM}$ calculations and the analysis of the active site in the crystal structures of persulfurated $\left(\mathrm{CsdA}_{\text {per }}\right)_{2}$ and $\left(\mathrm{CsdA}_{\text {per }}-\mathrm{CsdE}_{\text {per }}\right)_{2}$ demonstrate the existence of a electrostatic stabilization role for the Cys358 loop of CsdA (QHCAQ), the signature motif in SufS-like group II cysteine desulfurases that includes CsdA and SufS, and suggest stabilizing/catalytic roles for the conserved His119, His120, and the main chain and side chain of the less conserved Lys250* residue, in particular in stabilizing the developing negative charge on Cys358S $\delta$ and its subsequent reprotonation. The conservation of these critical residues enables the occasional crosstalk between systems without compromising their specificity. In group II Cys desulfurases in particular, the relative specificity of CsdA-CsdE and SufS-SufE pairs is generated by evolutionary divergence of secondary structure motifs (i.e., the elongation of strand $\beta 2$ and the C-terminal helix on the surface of CsdA) that leads to the optimization of the docking surface area of the Cys desulfurase for each system. In summary, group II cysteine desulfurases (and, 
possibly, group I cysteine desulfurases like IscS) have evolved towards versatile interaction surfaces that provide discrimination between their specific acceptors while deploying the precise chemical environment for the transpersulfuration reaction that mobilizes sulfane sulfur following a shared mechanism based in a highly conserved peptide motif near the catalytic Cys residues.

Scheme 1. Generic enzymatic mechanism proposed to explain the persulfuration of cysteine desulfurases the transpersulfuration of co-substrate sulfur acceptors.

Figure 1. X-ray crystal structure of persulfurated (CsdA-CsdE) $)_{2}$ complex. (A) ITC thermogram and binding isotherm for the interaction of CsdA with CsdE at $310 \mathrm{~K}$. In the upper panel, peaks indicate the heat released after each consecutive addition of CsdE into the CsdA protein solution. In the lower panel, the data were fit (continuous line) to a single binding model to calculate the thermodynamic parameters. Monomer molar concentrations were used for the fit. (B) Ribbon representation of the persulfurated (CsdA-CsdE $)_{2}$ heterotetramer. CsdA chains are shown in green and violet, and CsdE chains in yellow and slate blue. Side chains of PLP and persulfurated CsdA Cys358 and CsdE Cys61 are represented as space-filling models in CPK colors. (C) Structural basis for the conservation of the peptide signature motif of group II cysteine desulfurases. Interactions stabilizing the persulfidic $\mathrm{S}$ atoms in the $\mathrm{CsdA}_{\text {per }}-\mathrm{CsdE}_{\text {per }}$ complex (dashed lines).

Figure 2. Active site structures of (A) persulfurated $\left(\operatorname{CsdA}_{\text {per }}\right)_{2}$, (B) $S$-sulfated $(\mathrm{CsdA})_{2}$ and (C) persulfurated (Csd $\left.\mathrm{A}_{\text {per }}-\mathrm{CsdE}_{\text {per }}\right)_{2}$. Amino acid residues are represented in sticks and shown in CPK colors except carbon atoms; carbon atoms are colored in grey (Lys222-PLP), green (CsdA monomer in complex with CsdE), violet (opposite CsdA monomer), and cyan (CsdE). 
Interacting atoms relevant for the transpersulfuration reaction are connected with black dashed lines. Electron density maps contoured at $1 \sigma$ are overlaid on Lys222-PLP and the catalytic Cys residues (CsdA Cys358 and CsdE Cys61).

Figure 3. Conformational changes in bound $\mathrm{CsdE}_{\text {per }}$ facilitate transpersulfuration. (A) Superposition of the NMR structure of free CsdE (PDB 1NI7, in grey) ${ }^{30}$ onto the crystal structure of the persulfurated complex ( $\mathrm{CsdA}_{\mathrm{per}}$ in green, $\mathrm{CsdE}_{\text {per }}$ in cyan). The superposition highlights the concerted structural rearrangements that lead to the newly formed $\mathrm{H} 7$ (in bound $\mathrm{CsdE}_{\mathrm{per}}$ ) and the splitting of helix $\mathrm{H} 7$ (in free CsdE) into H8' and $\mathrm{H} 8$ in bound $\mathrm{CsdE}_{\text {per }}$ (blue arrow). The orientation between the new $\mathrm{H} 7, \mathrm{H} 8^{\prime}$ and $\mathrm{H} 8$ is approximately $85^{\circ}$ (blue arrow). These structural changes result in the swinging out of Cys61 from a semi-buried conformation in free CsdE to a fully extended conformation that allows the approach of the acceptor and donor Cys residues in the complex. (B) Superposition of the crystal structure of the persulfurated complex (CsdA $A_{\text {per }}$ in green, $\mathrm{CsdE}_{\text {per }}$ in cyan) with the heterodimer of the apo complex (PDB 4LW4; CsdA in white, CsdE in wheat ${ }^{20}$ with a traced Cys61. Helices H7-H8 of CsdE in the superimposed apo complex exhibit an intermediate conformation between free $\mathrm{CsdE}$ and bound $\mathrm{CsdE}_{\mathrm{per}}$. The distance between Cys61S $\delta$ and Cys358S $\delta$ in the persulfurated complex is shown as black dashed lines and that between Cys61S $\gamma$ and Cys358S $\gamma$ in the apo complex is shown in orange.

Figure 4. (CsdA-CsdE) $)_{2}$ interface probed by NMR. (A) ${ }^{1} \mathrm{H}^{-15} \mathrm{~N}$ HSQC spectra of $0.4 \mathrm{mM}$ uniformly labeled ${ }^{15} \mathrm{~N}-\mathrm{CsdE}$ either free in solution or upon addition of unlabeled CsdA to 1:0.2, 1:0.4 and 1:0.6 molar ratios. (B) Ribbon representation of the solution NMR CsdE structure (PDB 1NI7). Amino acids whose NH backbone correlation crosspeak volume in the HSQC spectrum decreases to a larger extent than the average upon CsdA addition are colored in red and boxed by red squares in (A). Cys61 is indicated in spheres ( $\mathrm{S}$ in yellow, $\mathrm{C}$ in white). (C) Plot of 
the decay rate of crosspeak volume (red circles) of CsdE residues upon CsdA addition, according to an exponential decay function $V=A \mathrm{e}^{-B x}$, where $V$ is the volume of an NH crosspeak, $A$ is a scaling factor, and $x$ is the CsdE:CsdA molar ratio. Blue crosses represent the fitting error on $B$. Residues with the fastest decay of the volume crosspeak (largest $B$ ) are annotated.

Figure 5. Catalytic residues involved in transpersulfuration across the (CsdA-CsdE) interface. (A) The persulfurated $\left(\mathrm{CsdA}_{\mathrm{per}}-\mathrm{CsdE}_{\mathrm{per}}\right)_{2}$ crystal structure, with both catalytic Cys residues as persulfides. (B) Insight into the active site from the minimized structure obtained at the M06-2X/6-31G**/MM level of theory, with Cys61 modeled with a thiolate side chain. The atoms belonging to the QM subsystem are shown as ball and sticks. Carbon atoms are depicted in cyan $(\mathrm{CsdE})$ and green $\left(\mathrm{CsdA}_{\text {per }}\right)$ in $(\mathrm{A})$ and dark olive green in $(\mathrm{B})$, with relevant interactions shown as dashed lines with their interatomic distances shown in $\AA$ (inter-S interactions are shown in blue).

Figure 6. Inter-sulfur distance fluctuations during MD simulations. Fluctuations of the distance (in $\AA$ ) between the S $\delta$ atom of CsdE Cys61 and the S $\gamma$ atom of the persulfurated CsdA Cys358 observed during $50 \mathrm{~ns}$ of MD simulation. CsdE Cys61S $\delta$ was modeled in the ionized form (thiolate), as this is the physiologically relevant form (experimental $\mathrm{p} K_{a}=6.5$ ).

Figure 7. Transition structure for the transpersulfuration reaction. Ball-and-stick representation of the transition structure observed during MD simulations of the (Csd $\left.\mathrm{A}_{\text {per }}-\mathrm{CsdE}\right)_{2}$ structure. Color codes for carbon atoms are cyan (CsdE) and green and violet (CsdA subunits). The $\mathrm{S} \gamma$ atom is shown on the path between the donor CsdA Cys358S $\delta$ (modeled initially as a thiol function) and the acceptor CsdE Cys61S $\delta$ (modeled initially as a thiolate), with inter-sulfur interactions depicted as dashed lines with distance labels in blue, and all other interactions and 
distances in black. It is noteworthy the participation of His119 and Lys250* $(*$ denotes the other subunit) in stabilizing the negative charge that develops on CsdA Cys358S $\delta$ as the transpersulfuration progresses and ultimately in its reprotonation. 
Table 1. X-ray data collection and refinement statistics.

\begin{tabular}{|c|c|c|}
\hline & $\left(\mathrm{CsdA}_{\mathrm{per}}\right)_{2}$ & $\left(\mathrm{CsdA}_{\text {per }}-\mathrm{CsdE}_{\mathrm{per}}\right)_{2}$ \\
\hline Wavelength $(\AA)$ & 0.87260 & 0.98011 \\
\hline \multirow[t]{2}{*}{ Resolution $(\AA)$} & $36.7-2.38$ & $48.3-2.49$ \\
\hline & $(2.47-2.38)$ & $(2.63-2.49)$ \\
\hline Space group & $P 2_{1} 2_{1} 2_{1}$ & $P 2_{1} 2_{1} 2_{1}$ \\
\hline \multirow[t]{2}{*}{ Unit cell $\left(\AA,{ }^{\circ}\right)$} & 58.399 .5141 .7 & 74.8115 .1604 .9 \\
\hline & 909090 & 909090 \\
\hline Total reflections & $124,259(12,453)$ & $625,506(64,000)$ \\
\hline Unique reflections & $29,943(3051)$ & $178,722(23,193)$ \\
\hline Multiplicity & $4.1(4.1)$ & $3.5(2.8)$ \\
\hline Completeness (\%) & $89.40(91.51)$ & $97.90(88.30)$ \\
\hline Mean $I / \sigma(I)$ & $11.13(2.40)$ & $7.40(1.80)$ \\
\hline Wilson $B$-factor $\left(\AA^{2}\right)$ & 22.65 & 49.53 \\
\hline $\mathrm{R}_{\text {merge }}$ & $0.176(0.736)$ & $0.127(0.553)$ \\
\hline $\mathrm{R}_{\text {meas }}$ & 0.1966 & 0.1490 \\
\hline $\mathrm{CC}_{1 / 2}$ & $0.990(0.703)$ & $0.989(0.581)$ \\
\hline $\mathrm{CC}^{*}$ & $0.997(0.909)$ & $0.996(0.815)$ \\
\hline $\mathrm{R}_{\text {work }}$ & $0.1669(0.2306)$ & $0.2532(0.3566)$ \\
\hline $\mathrm{R}_{\text {free }}$ & $0.2245(0.3001)$ & $0.2768(0.4141)$ \\
\hline$\#$ of non- $\mathrm{H}$ atoms & 6525 & 34,325 \\
\hline Macromolecules & 6139 & 32,886 \\
\hline Ligands & 87 & 418 \\
\hline Water & 299 & 1021 \\
\hline Protein residues & 800 & 4296 \\
\hline
\end{tabular}




\begin{tabular}{|l|l|l|}
\hline RMSD (bonds) $(\AA)$ & 0.004 & 0.010 \\
\hline RMSD (angles) $\left(^{\circ}\right)$ & 0.77 & 1.20 \\
\hline Ramachandran favored (\%) & 97.00 & 96.00 \\
\hline Ramachandran outliers (\%) & 0.00 & 0.68 \\
\hline Clashscore & 8.29 & 8.09 \\
\hline Average $B$-factor & 30.50 & 68.70 \\
\hline Macromolecules & 29.80 & 69.70 \\
\hline Ligands & 62.10 & 59.30 \\
\hline Solvent & 33.70 & 40.40 \\
\hline PDB ID & $5 \mathrm{FT} 5$ & $5 \mathrm{FT} 8$ \\
\hline
\end{tabular}

\section{ASSOCIATED CONTENT}

Supporting Information. Experimental procedures, X-ray diffraction data processing, structure refinement tables, ITC, NMR, SAXS, more detailed comparisons of the apo and persulfurated (CsdA-CsdE $)_{2}$ complex, details of the computational chemistry calculations and the optimized geometry of the QM optimized subsystem, electron density maps supporting $\mathrm{CsdE}_{\text {per }}$ conformational changes upon complexation, and comparison of $\left(\mathrm{CsdA}_{\mathrm{per}}-\mathrm{CsdE}_{\mathrm{per}}\right)_{2}$ with (IscS-IscU/TusA) $)_{2}$. This material is available free of charge via the Internet at http://pubs.acs.org.

\section{AUTHOR INFORMATION}

\section{Corresponding Author}

* M. Cristina Vega. cvega at cib.csic.es, cristina.vega at strubicib.org.

\section{Present Addresses}

$\dagger$ Structural Biology, CIC bioGUNE, Derio-Bizkaia, Spain 


\section{Author Contributions}

The manuscript was written through contributions of all authors. All authors have given approval to the final version of the manuscript.

\section{Funding Sources}

This work was funded by Spanish institutional grants BFU2008-02372/BMC, CSD 2006-23, and BFU2011-22588 to M.C., CTQ2012-36253-C03-03 to I.T., and BFU2010-22266-C02-02 and CTQ2015-66206-C2-2-R to M.C.V. Further support for this work was obtained from the Generalitat Valenciana (ACOMP/2015/239 to I.T.) and from the European Commission FP7 ComplexINC grant (contract no. 279039) to M.C.V.

\section{Notes}

The authors declare no competing financial interests.

\section{ACKNOWLEDGMENT}

We acknowledge the ESRF for provision of synchrotron radiation at ID23-1, ID23-2, ID14-1, and BM29 BioSAXS beam lines. E.P.S. and M.L.E. acknowledge the support of the Ph.D. program in Structure and Function of Proteins of the Universitat Autònoma de Barcelona (UAB) and the Ph.D. program in Biochemistry, Molecular Biology and Biomedicine of the Universidad Complutense de Madrid (UCM), respectively.

\footnotetext{
ABBREVIATIONS

AUC, analytical ultracentrifuge; CSD, cysteine desulfurase; ISC, iron-sulfur cluster; ITC, isothermal titration calorimetry; PLP, pyridoxal 5'-phosphate; RMSD, root-mean-square distance; SUF, sulfur utilization.
} 


\section{REFERENCES}

(1) Kessler, D. FEMS Microbiol Rev 2006, 30, 825-840.

(2) Mueller, E. G. Nat Chem Biol 2006, 2, 185-194.

(3) Begley, T. P.; Xi, J.; Kinsland, C.; Taylor, S.; McLafferty, F. Curr Opin Chem Biol 1999, $3,623-629$.

(4) Marquet, A. Curr Opin Chem Biol 2001, 5, 541-549.

(5) Ayala-Castro, C.; Saini, A.; Outten, F. W. Microbiol Mol Biol Rev 2008, 72, 110-25, table of contents.

(6) Fontecave, M.; Ollagnier-de-Choudens, S. Arch Biochem Biophys 2008, 474, 226-237.

(7) Johnson, D. C.; Dean, D. R.; Smith, A. D.; Johnson, M. K. Annu Rev Biochem 2005, 74, 247-281.

(8) Fontecave, M.; Choudens, S. O.; Py, B.; Barras, F. J Biol Inorg Chem 2005, 10, 713-721.

(9) Loiseau, L.; Ollagnier-de Choudens, S.; Lascoux, D.; Forest, E.; Fontecave, M.; Barras, F. J Biol Chem 2005, 280, 26760-26769.

(10) Trotter, V.; Vinella, D.; Loiseau, L.; Ollagnier de Choudens, S.; Fontecave, M.; Barras, F. Mol Microbiol 2009, 74, 1527-1542.

(11) Miyauchi, K.; Kimura, S.; Suzuki, T. Nat Chem Biol 2013, 9, 105-111.

(12) López-Estepa, M.; Ardá, A.; Savko, M.; Round, A.; Shepard, W. E.; Bruix, M.; Coll, M.; Fernández, F. J.; Jiménez-Barbero, J.; Vega, M. C. PLoS ONE 2015, 10, e0118606.

(13) Mihara, H.; Esaki, N. Appl Microbiol Biotechnol 2002, 60, 12-23.

(14) Zheng, L.; Cash, V. L.; Flint, D. H.; Dean, D. R. J Biol Chem 1998, 273, 13264-13272.

(15) Mihara, H.; Maeda, M.; Fujii, T.; Kurihara, T.; Hata, Y.; Esaki, N. J Biol Chem 1999, $274,14768-14772$.

(16) Pan, J.; Carroll, K. S. ACS Chem Biol 2013, 8, 1110-1116.

(17) Shi, R.; Proteau, A.; Villarroya, M.; Moukadiri, I.; Zhang, L.; Trempe, J. F.; Matte, A.; Armengod, M. E.; Cygler, M. PLoS Biol 2010, 8, e1000354.

(18) Marinoni, E. N.; de Oliveira, J. S.; Nicolet, Y.; Raulfs, E. C.; Amara, P.; Dean, D. R.; Fontecilla-Camps, J. C. Angew Chem Int Ed Engl 2012, 51, 5439-5442.

(19) Lima, C. D. J Mol Biol 2002, 315, 1199-1208.

(20) Kim, S.; Park, S. J Biol Chem 2013, 288, 27172-27180.

(21) Kabsch, W. Acta Crystallogr D Biol Crystallogr 2010, 66, 125-132.

Con formato: Inglés (Estados Unidos) 
(22) Evans, P. R. Acta Crystallogr D Biol Crystallogr 2011, 67, 282-292.

(23) McCoy, A. J.; Grosse-Kunstleve, R. W.; Adams, P. D.; Winn, M. D.; Storoni, L. C.; Read, R. J. J Appl Crystallogr 2007, 40, 658-674.

(24) Afonine, P. V.; Grosse-Kunstleve, R. W.; Echols, N.; Headd, J. J.; Moriarty, N. W.; Mustyakimov, M.; Terwilliger, T. C.; Urzhumtsev, A.; Zwart, P. H.; Adams, P. D. Acta Crystallogr D Biol Crystallogr 2012, 68, 352-367.

(25) Emsley, P.; Lohkamp, B.; Scott, W. G.; Cowtan, K. Acta Crystallogr D Biol Crystallogr 2010, 66, 486-501.

(26) Chen, V. B.; Arendall, W. B.; Headd, J. J.; Keedy, D. A.; Immormino, R. M.; Kapral, G. J.; Murray, L. W.; Richardson, J. S.; Richardson, D. C. Acta Crystallogr D Biol Crystallogr 2010, 66, 12-21.

(27) Vranken, W. F.; Boucher, W.; Stevens, T. J.; Fogh, R. H.; Pajon, A.; Llinas, M.; Ulrich, E. L.; Markley, J. L.; Ionides, J.; Laue, E. D. Proteins 2005, 59, 687-696.

(28) Grzesiek, S.; Bax, A. Journal of Magnetic Resonance (1969) 1992, 99, 201-207.

(29) Grzesiek, S.; Bax, A. J Am Chem Soc 1992, 114, 6291-6293.

(30) Liu, G.; Li, Z.; Chiang, Y.; Acton, T.; Montelione, G. T.; Murray, D.; Szyperski, T. Protein Sci 2005, 14, 1597-1608.

(31) Case, D. A.; Berryman, J. T.; Betz, R. M.; Cerutti, D. S.; Cheatham, III, T. E.; Darden, T. A.; Duke, R. E.; Giese, T. J.; Gohlke, H.; Goetz, A. W.; Homeyer, N.; Izadi, S.; Janowski, P.; Kaus, J.; Kovalenko, A.; Lee, T. S.; LeGrand, S.; Li, P.; Luchko, T.; Luo, R.; Mandej, B.; Merz, K. M. The Amber Molecular Dynamics Package; University of California: San Francisco, 2015.

(32) Phillips, J. C.; Braun, R.; Wang, W.; Gumbart, J.; Tajkhorshid, E.; Villa, E.; Chipot, C.; Skeel, R. D.; Kalé, L.; Schulten, K. J Comput Chem 2005, 26, 1781-1802.

(33) Field, M. J.; Bash, P. A.; Karplus, M. J Comput Chem 1990, 11, 700-733.

(34) J. Frisch, M.; W. Trucks, G.; B. Schlegel, H.; E. Scuseria, G.; A. Robb, M.; R. Cheeseman, J.; Scalmani, G.; Barone, V.; Mennucci, B.; A. Petersson, G.; Nakatsuji, H.; Caricato, M.; Li, X.; P. Hratchian, H.; F. Izmaylov, A.; Bloino, J.; Zheng, G.; L. Sonnenberg, J.; Hada, M.; Ehara, M.; Toyota, K.; Fukuda, R.; Hasegawa, J.; Ishida, M.; Nakajima, T.; Honda, Y.; Kitao, O.; Nakai, H.; Vreven, T.; Montgomery, J., J. A.; E. Peralta, J.; Ogliaro, F.; Bearpark, M.; J. Heyd, J.; Brothers, E.; N. Kudin, K.; N. Staroverov, V.; Kobayashi, R.; Normand, J.; Raghavachari, K.; Rendell, A.; C. Burant, J.; S. Iyengar, S.; Tomasi, J.; Cossi, M.; Rega, N.; M. Millam, J.; Klene, M.; E. Knox, J.; B. Cross, J.; Bakken, V.; Adamo, C.; Jaramillo, J.; Gomperts, R.; E. Stratmann, R.; Yazyev, O.; J. Austin, A.; Cammi, R.; Pomelli, C.; W. Ochterski, J.; L. Martin, R.; Morokuma, K.; G. Zakrzewski, V.; A. Voth, G.; Salvador, P.; J. Dannenberg, J.; Dapprich, S.; D. Daniels, A.; Farkas, Ö.; B. Foresman, J.; V. Ortiz, J.; Cioslowski, J.; J. Fox, D. Gaussian 09 $\{R\}$ evision $\{D\} .01$; Gaussian Inc., 2009. 
(35) Zhao, Y.; Truhlar, D. G. Theor Chem Acc 2008, 120, 215-241.

(36) Aranda, J.; Zinovjev, K.; Roca, M.; Tuñón, I. J Am Chem Soc 2014, 136, 16227-16239.

(37) Krzemińska, A.; Paneth, P.; Moliner, V.; Świderek, K. J Phys Chem B 2015, 119, 917927.

(38) Field, M. J.; Albe, M.; Bret, C.; Flavien, P. M.; Thomas, A. J Comput Chem 2000.

(39) Singh, U. C.; Kollman, P. A. J Comput Chem 1986, 7, 718-730.

(40) Prischi, F.; Konarev, P. V.; Iannuzzi, C.; Pastore, C.; Adinolfi, S.; Martin, S. R.; Svergun, D. I.; Pastore, A. Nat Commun 2010, 1, 95.

(41) Singh, H.; Dai, Y.; Outten, F. W.; Busenlehner, L. S. J Biol Chem 2013, 288, 3618936200 .

(42) Selbach, B.; Earles, E.; Dos Santos, P. C. Biochemistry 2010, 49, 8794-8802.

(43) Urbina, H. D.; Silberg, J. J.; Hoff, K. G.; Vickery, L. E. J Biol Chem 2001, 276, 4452144526.

Con formato: Inglés (Estados Unidos)

(44) Bolstad, H. M.; Botelho, D. J.; Wood, M. J. J Proteome Res 2010, 9, 5358-5369. 


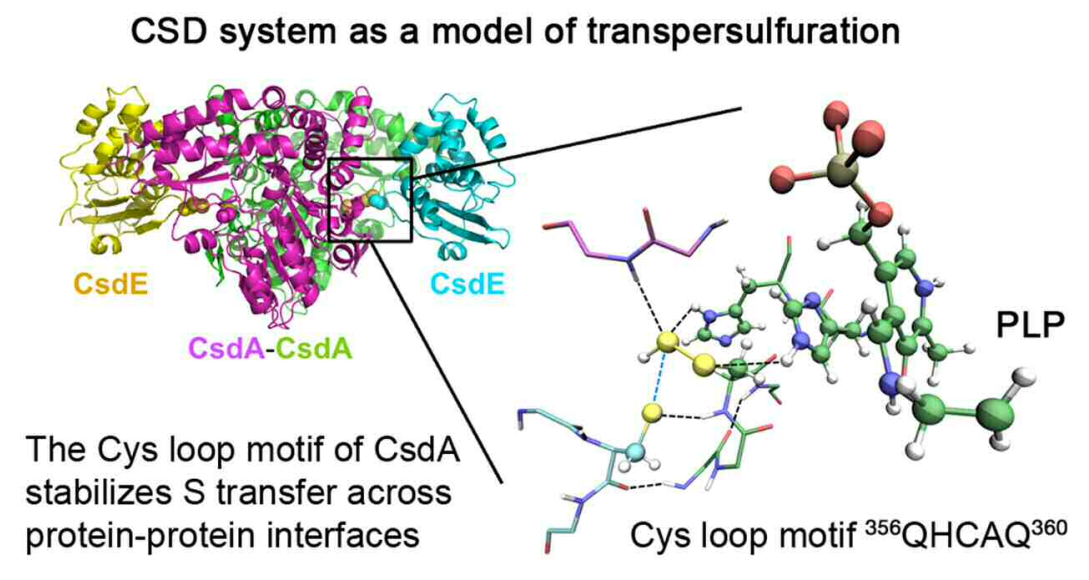

For the Table of Contents only 